\title{
Evidence of Macular Pigment in the Central Macula in Albinism
}

Yulia Wolfson, M.D. ${ }^{1}$, Emily Fletcher, M.D. ${ }^{1,2}$, Rupert W. Strauss, M.D. ${ }^{1,3}$, Hendrik P. N. Scholl, M.D. ${ }^{1}$

1. Ophthalmology, Johns Hopkins University, Baltimore, MD, United States

2. Ophthalmology, Gloucestershire NHS Trust, Gloucester, United Kingdom

3. Ophthalmology, Medical University Graz, Graz, Austria

Corresponding author: Hendrik P. N. Scholl. 600 N. Wolfe Street, Maumenee 748, Baltimore, MD 21287-9277, USA. Office: 410-614-6908; fax: 410-614-2186.

hscholl1@jhmi.edu 


\section{Evidence of Macular Pigment in the Central Macula in Albinism}

\section{Abstract}

3 Purpose: Albinism represents a spectrum of disorders with diminished to absent

4 amounts of melanin pigmentation including the posterior segment of the eye. Macular

5 pigment (MP) consists of two main carotenoids, lutein and zeaxanthin, concentrated in

6 the macula. MP serves as blue light absorbent, antioxidant, and may reduce chromatic

7 aberration and glare. It remains unclear if albinos have detectable MP. The purpose was

8 to investigate the distribution of MP in albino patients with psychophysical and imaging

9 techniques.

10 Methods: MP was measured at the eccentricity of 0.5 degrees by heterochromatic

11 flicker perimetry (QuantifEye®; Tinsley Precision Instruments Ltd.) or by scanning laser

12 ophthalmoscopy (MPOD module, MultiColor Spectralis®, Heidelberg Engineering,

13 Heidelberg, Germany) in four enrolled albino patients, who were also investigated with

14 multimodal ophthalmic imaging.

15 Results: Visual acuity ranged from $20 / 32$ to $20 / 125$, nystagmus was present in three

16 patients, and all patients showed typical foveal hypoplasia on fundus exam and optical

17 coherence tomography. Fundus autofluorescence (FAF) demonstrated various degrees

18 of central FAF signal attenuation. Genetic testing was available in three patients and

19 confirmed the diagnosis. Measurable amounts of MP were detected in all four patients

20 and ranged from 0.05 to 0.24 , which is below the normal expected range.

21 Conclusions: We conclude that MP can be demonstrated and measured in albinos.

22 Further studies are needed to investigate MP accumulation following carotenoid

23 supplementation.

24 Key words: albinism, macular pigment, foveal hypoplasia, lutein, zeaxanthin, diet 
25 supplementation

26 


\section{Introduction}

28 Ocular and oculocutaneous albinism represent a spectrum of disorders with absent or

29 significantly diminished amount of melanin either across different body tissues (OCA1

30 and OCA2), or exclusively in eye tissues (OA1).(Summers, 2009) Clinical findings and

31 visual performance show significant variability without obvious phenotype-genotype

32 correlation.(Gargiulo et al., 2011) Typically, the ocular phenotype includes iris

33 transillumination, foveal hypoplasia, nystagmus, reduced best-corrected visual acuity,

34 refractive errors, photosensitivity, and abnormal development of the visual pathways with

35 characteristic abnormal routing of ganglion cell axons in the chiasma, resulting in

36 abnormal pattern visually evoked potentials.(Dorey et al., 2003) Current treatment

37 options are limited to low vision aids. Previously correlation was found between the

38 amount of melanin fundus pigmentation and visual function in albino patients.(Summers,

39 1996)

40 The macular pigment (MP) consists of two main carotenoids, lutein and zeaxanthin,

41 which are concentrated in the macula.(Whitehead et al., 2006) MP is hypothesized to

42 function as a protector by absorbing blue light incident on the retina, thereby reducing

43 photo-oxidative stress to the underlying photoreceptors. It is also thought to improve

44 visual function via reduction of chromatic aberration and glare.(Wooten and Hammond,

45 2002) The only report on macular pigment in albino patients failed to demonstrate any

46 MP.(Abadi and Cox, 1992) The MP would be a hypothetical candidate to improve visual

47 performance, simply by increasing pigmentation, reducing light scatter and thus glare

48 sensitivity. MPOD normal range in US population has been estimated to be $0.24-0.45$.

49 Even in normal subjects, supplementation and an increase in the macular pigment

50 optical density (MPOD) could be correlated with increased visual performance in

51 randomized, placebo-controlled studies. (Nolan et al., 2011, Trieschmann et al., 2007, 
52 van den Berg et al., 2009) As this pigment is not produced in the retina, but is absorbed

53 via diet, it can be manipulated by diet and supplementation and thereby providing an

54 opportunity to positively impact visual performance. It needs to be determined if MP is

55 detectable at all in albinism.

56 In this case series, we evaluated 4 albino patients and performed measurements of MP:

57 in three patients by heterochromatic flicker photometry (HFP), and in one patient with the

58 novel MPOD module of the Heidelberg MultiColor Spectralis ${ }^{\circledR}$.

\section{Methods}

60 Four albino patients were enrolled. Multimodal retinal imaging was performed. MPOD

61 measurements were performed with the QuantifEye® device in 3 albino patients, and an

62 additional patient underwent MPOD evaluation with the new MPOD module of the

63 Heidelberg Multicolor Spectralis $\AA$. The study followed the tenets of the Declaration of

64 Helsinki and was approved by the local Ethics Review Board.

65 The most widely used technique to assess the MPOD is the HFP method: A small

66 (usually about 1 degree) visual stimulus with alternating blue (wavelength $465 \mathrm{~nm}$,

67 maximally absorbed by MP) and green (wavelength $530 \mathrm{~nm}$, not significantly absorbed

68 by MP) light is presented. We used the QuantifEye® device (QuantifEye®; MPS 9000

69 series: Tinsley Precision Instruments Ltd., Croydon, Essex, UK) for HFP. The device

70 was first described in 2009 (van der Veen et al., 2009a), and showed good repeatability

71 ( $r=0.97)$, and produced similar results to retinal reflectometry(van der Veen et al., 2009b)

72 and MultiColor Spectralis® HRA(Dennison et al., 2013). The device provides the option

73 to either test only the central MPOD ( 0.5 degrees eccentricity) or perform both the

74 central and the peripheral test of MPOD (performed at 6 degrees eccentricity). In

75 resemblance to the visual field test, during MPOD testing the patient is required to press 
76 the button, once he notices the appearance of the flicker at the stimulus spot. While

77 performing central measurement, the flicker stimulus is presented centrally at the fixation

78 point, and for peripheral measurement, the flicker is presented at the same central

79 location, however the fixation point is moved to the periphery of the presented field.

80 Peripheral measurement can be challenging due to the required peripheral fixation. If the

81 peripheral measurement is not performed for any reason, the central measurement is

82 estimated based on age-adjusted data, taking into account the "normal yellowing" of the

83 natural human lens. We attempted both, central and peripheral testing in our patients.

84 The measurements were performed with non-dilated pupils, in dim light settings and with

85 habitual correction, based on manufacturer's instructions.

86 The MPOD module of Spectralis $\AA$ uses the MultiColor Spectralis $\AA$ platform instrument

87 as the basis. In difference to the BluePeak system used to acquire blue AF images on

88 the (standard) Heidelberg Spectralis $\AA$, it is equipped with an additional green laser,

89 resulting in the ability to acquire infrared, green, and blue reflectance images from the

90 retina simultaneously. For the MP measurements, the fluorescence detection is used to

91 measure MP. The built-in software module performs the final MPOD calculation (as

92 described by Trieschmann et al.(Trieschmann et al., 2006)) Once the measurement is

93 performed, the average MPOD can be calculated at any eccentricity covered by the

94 original scan (5 degrees) by the in-built software. In our study we report 0.5 eccentricity

95 measurements, similar to the eccentricity used by the QuantifEye $\AA$ device. The

96 measurements were performed with dilated pupils, in dim light settings, and with optimal

97 visualization of the anatomical features on the preceding red-free scan, utilizing

98 optimization of focal distance and magnification. 


\section{Results:}

101 The clinical characteristics of the 4 albino patients are summarized below (only positive

102 and disease-specific findings are noted in the descriptions below, while normal finding

103 are omitted):

104 Patient 1: forty two-year-old male with sporadic oculocutaneous albinism. His best-

105 corrected visual acuity was $20 / 63$ in the right eye and $20 / 50$ in the left; no nystagmus but

106 mild iris transillumination were noted on ocular exam. On dilated fundus examination a

107 low-pigmented fundus and absence of the foveal reflex were noted. Foveal hypoplasia

108 was confirmed by OCT (Figure 1, B1). Molecular diagnosis is not available for this

109 patient. The macular pigment measured by QuantifEye was 0.05 in the right eye, with

110 reliable central and peripheral measurements, and not measurable in the left eye due to

111 unreliable central and peripheral measurements.

112 Patient 2: Fifty-year-old male with oculocutaneous albinism. His best-corrected visual

113 acuity on exam was 20/63 in the right and 20/50 in the left eye with hyperopic astigmatic

114 correction. He had pendular nystagmus, moderate iris transillumination and lack of the

115 foveal reflex. OCT scan confirmed foveal hypoplasia in both eyes (Figure 1, B2). Genetic

116 testing was consistent with OCA1 (TYR gene Compound Heterozygous Mutation:

117 c.61C>T (p.Pro21Ser) in exon 1, c.1037-7T>A (IVS2-7T>A) in exon 3). The macular

118 pigment measured by QuantifEye was 0.14 in the right eye, with reliable central but

119 unreliable peripheral measurements, and not measurable in the left eye due to

120 significant nystagmus.

121 Patient 3: sixty five-year-old male, with best-corrected visual acuity of $20 / 80$ in the right

122 and 20/50 in the left eye, pendular nystagmus, absence of iris transillumination and

123 foveal hypoplasia in both eyes as detected by dilated fundus examination and confirmed 
124 by OCT (Figure 1, B3). Additional clinical findings included bilateral hypermetropia,

125 alternating exotropia, and nuclear sclerosis of the lens in the right eye. He had a positive

126 family history of albinism: two affected siblings, daughter and granddaughter. $\mathrm{He}$

127 reported no history of consanguinity in the family. Genetic testing was consistent with

128 OCA2 (Single Heterozygous Mutation: c.1103C>T (p.Ala368Val) in exon 10). Macular

129 pigment measured by QuantifEye was 0.12 in the right eye, with reliable central and

130 peripheral measurements, and 0.24 in the left eye with reliable central and unreliable

131 peripheral measurements.

132 Patient 4: forty-year-old male with oculocutaneous albinism. He was emmetropic, and

133 his uncorrected visual acuity was $20 / 40$ in the right eye and $20 / 32$ in the left. There was

134 no nystagmus and no iris transillumination on ocular exam. Foveal hypoplasia was

135 detected in both eyes on dilated fundus exam, and confirmed by OCT (Figure 1, B4).

136 Homozygous p.Val443lle:c.1327G>A mutation was detected, consistent with the

137 diagnosis of OCA2. Macular pigment measured by MultiColor was 0.15 in the right eye,

138 and 0.18 in the left eye. (Figure 2)

139 Figure 1 shows retinal images of the right eye of each patient. As can be observed on

140 fundus autofluorescence imaging (Figure 1, C1-C4), different degrees of foveal AF

141 signal attenuation due to absorbance by the MP can be detected: while in patients 1 and

142 2, there appears to be only a weak, but definite central attenuation of the AF signal,

143 patient 4, and even more so, patient 3 demonstrated quite normal distribution of AF

144 signal with central AF attenuation. OCT scans of all 4 patients demonstrated foveal

145 hypoplasia with complete absence of the foveal pit and presence of all inner retinal

146 layers throughout the fovea (Figure 1, B1-B4).

147 MP measurements with the QuantifEye ${ }^{\circledR}$ were technically possible in 5 out of 6 eyes of 
148 Patients 1,2 and 3. Central MPOD measurements were possible in all 5 eyes, however

149 appeared unreliable (based on a QuantifEye® software output) in 1 out of 5 eyes (left

150 eye of Patient 1). The peripheral MPOD measurements were technically possible in only

1512 out of 4 eyes (right eye of Patient 1 and right eye of Patient 3). In Patient 3, MPOD in

152 the right eye was 0.12 , as calculated from central and peripheral measurements. In the

153 left eye, the peripheral measurement of MPOD appeared to be unreliable, and therefore

154 the final MPOD was based on age-matched data, and was estimated to be 0.24 . We

155 believe that the inter-eye discrepancy of MPOD in Patient 3 can be explained by

156 presence of nuclear cataract in this patient. In Patient 4, the MPOD measurement was

157 performed by means of the MultiColor Spectralis ${ }^{\circledR}$, and measured 0.15 in the right eye

158 and 0.18 in the left eye. The differential map of blue and green fundus autofluorescence

159 clearly shows accumulation of MP in the central retina (Figure 2).

160 Discussion

161 The existence and distribution of MP in albino patients has not been previously

162 evaluated by currently available techniques: one single report from 1992 concluded that

163 MPOD is not measurable. In one recent report of MPOD in an oculocutaneous albinism

164 patient, the value of MPOD was found to be 0.1 , as measured by HFP

165 technique.(Putnam and Bland, 2014) The Macular Pigment Reflectometer (MPR) (van

166 de Kraats et al., 2006) device would offer an "objective" method; however there are

167 limitations to use the device in certain conditions (such as albinism) due to its low

168 reliability in performing measurements in absence of choroidal and retinal melanin. The

169 MPOD measurement with the novel MPOD module of the MultiColor Spectralis $\AA$

170 provides objective measurements of the macular pigment. In our patient, a low but

171 measurable amount of MP was detected. The spatial MP profile showed an elevated 
172 central plateau as opposed to a more pronounced central or pericentral peak that is

173 usually demonstrated in normal retinas. It must be noted that the shortcomings of the

174 HFP psychophysical technique for MP measurement are possibly further aggravated by

175 the impaired central visual acuity, nystagmus and poor fixation in albino patients, and

176 can lead to substantial MPOD measurement artifacts in albino population.

177 Our data suggest that absence of the normal foveal pit development does not preclude

178 MP deposition and that the fovea is still being "recognized", at least to some degree, by

179 MP molecule transport mechanisms. We detected low levels of MP in four albino

180 patients; however clinical significance of such low levels regarding their impact on visual

181 performance remains unclear. The forthcoming LUVIA (LUtein for Vision in Albinism)

182 clinical trial will investigate MP accumulation following carotenoids supplementation and

183 its consequences for visual performance in albino patients (ClinicalTrials.gov Identifier:

184 NCT02200263).

185 In conclusion, we demonstrate measurable (although low) levels of MP in albino

186 patients, ranging from 0.05 to 0.24 . Further studies aiming to provide more cross-

187 sectional data on MPOD distribution as well as a hypothesized increase in MPOD

188 following carotenoid supplementation in this population may be warranted.

\section{Acknowledgement}

190 The study was supported by the Ocular Albinism Research Fund (Clark Enterprises

191 Inc.). Dr. Hendrik Scholl is supported by the Foundation Fighting Blindness Clinical

192 Research Institute (FFB CRI) and a grant to FFB CRI by the U.S. Department of

193 Defense USAMRMC TATRC, Fort Meade, Maryland (grant numbers W81-XWH-07-1-

1940720 and W81XWH-09-2-0189); The Shulsky Foundation, New York, NY; Unrestricted

195 grant to the Wilmer Eye Institute from Research to Prevent Blindness; Baylor-Johns 
196 Hopkins Center for Mendelian Genetics (National Human Genome Research Institute,

$197 \mathrm{NHGRI/NIH;}$; Identification number: 1U54HG006542-01). H.P.N.S. is the Dr. Frieda

198 Derdeyn Bambas Professor of Ophthalmology

199

200

201

202

203

204

205

206

207

208

209

210

211

212

213

214

215

216

217

218

219

220

221

222

223

224

225

226

227

\section{References:}

ABADI, R. V. \& COX, M. J. 1992. The distribution of macular pigment in human albinos. Invest Ophthalmol Vis Sci, 33, 494-7.

DENNISON, J. L., STACK, J., BEATTY, S. \& NOLAN, J. M. 2013. Concordance of macular pigment measurements obtained using customized heterochromatic flicker photometry, dual-wavelength autofluorescence, and single-wavelength reflectance. Exp Eye Res, 116, 190-8.

DOREY, S. E., NEVEU, M. M., BURTON, L. C., SLOPER, J. J. \& HOLDER, G. E. 2003. The clinical features of albinism and their correlation with visual evoked potentials. Br J Ophthalmol, 87, 767-72.

GARGIULO, A., TESTA, F., ROSSI, S., DI IORIO, V., FECAROTTA, S., DE BERARDINIS, T., IOVINE, A., MAGLI, A., SIGNORINI, S., FAZZI, E., GALANTUOMO, M. S., FOSSARELLO, M., MONTEFUSCO, S., CICCODICOLA, A., NERI, A., MACALUSO, C., SIMONELLI, F. \& SURACE, E. M. 2011. Molecular and clinical characterization of albinism in a large cohort of Italian patients. Invest Ophthalmol Vis Sci, 52, 1281-9.

NOLAN, J. M., LOUGHMAN, J., AKKALI, M. C., STACK, J., SCANLON, G., DAVISON, P. \& BEATTY, S. 2011. The impact of macular pigment augmentation on visual performance in normal subjects: COMPASS. Vision Res, 51, 459-69.

PUTNAM, C. M. \& BLAND, P. J. 2014. Macular pigment optical density spatial distribution measured in a subject with oculocutaneous albinism. J Optom, 7, 2415 .

SUMMERS, C. G. 1996. Vision in albinism. Trans Am Ophthalmol Soc, 94, 1095-155.

SUMMERS, C. G. 2009. Albinism: classification, clinical characteristics, and recent findings. Optom Vis Sci, 86, 659-62.

TRIESCHMANN, M., BEATTY, S., NOLAN, J. M., HENSE, H. W., HEIMES, B., AUSTERMANN, U., FOBKER, M. \& PAULEIKHOFF, D. 2007. Changes in macular pigment optical density and serum concentrations of its constituent carotenoids following supplemental lutein and zeaxanthin: the LUNA study. Exp Eye Res, 84, 718-28. 
TRIESCHMANN, M., HEIMES, B., HENSE, H. W. \& PAULEIKHOFF, D. 2006. Macular pigment optical density measurement in autofluorescence imaging: comparison of one- and two-wavelength methods. Graefes Arch Clin Exp Ophthalmol, 244, 1565-74.

VAN DE KRAATS, J., BERENDSCHOT, T. T., VALEN, S. \& VAN NORREN, D. 2006. Fast assessment of the central macular pigment density with natural pupil using the macular pigment reflectometer. J Biomed Opt, 11, 064031.

VAN DEN BERG, T. J., FRANSSEN, L. \& COPPENS, J. E. 2009. Straylight in the human eye: testing objectivity and optical character of the psychophysical measurement. Ophthalmic Physiol Opt, 29, 345-50.

VAN DER VEEN, R. L., BERENDSCHOT, T. T., HENDRIKSE, F., CARDEN, D., MAKRIDAKI, M. \& MURRAY, I. J. 2009a. A new desktop instrument for measuring macular pigment optical density based on a novel technique for setting flicker thresholds. Ophthalmic Physiol Opt, 29, 127-37.

VAN DER VEEN, R. L., BERENDSCHOT, T. T., MAKRIDAKI, M., HENDRIKSE, F., CARDEN, D. \& MURRAY, I. J. 2009b. Correspondence between retinal reflectometry and a flicker-based technique in the measurement of macular pigment spatial profiles. J Biomed Opt, 14, 064046.

WHITEHEAD, A. J., MARES, J. A. \& DANIS, R. P. 2006. Macular pigment: a review of current knowledge. Arch Ophthalmol, 124, 1038-45.

WOOTEN, B. R. \& HAMMOND, B. R. 2002. Macular pigment: influences on visual acuity and visibility. Prog Retin Eye Res, 21, 225-40. 

autofluorescence (FAF) images (Spectralis ${ }^{\circledR}$, Heidelberg Engineering). Left column (A1-A4) represents red free reflectance images of Patient 1-4 correspondingly. foveal OCT B-scans of Patients 1-4 correspondingly showing foveal hypoplasia in all 4 patients.

260 Right column (C1-C4) shows FAF scans. The FAF images (488nm, HRA2, Heidelberg Engineering; acquired with 100\% laser power and $90 / 91 \%$ sensitivity settings) show topographic distribution of autofluorescent (AF) signal in the right eye of Patients 1-4, correspondingly. Attenuation of AF signal in the foveal center, which is usually attributed to presence of macular pigment, can be observed in all patients, however to a different degree. (Symmetrical findings were observed in the contralateral eye of each patient).

MPOD values were $0.05,0.14$, and 0.12 for Patients $1-3$, correspondingly, as measured by QuantifEye ${ }^{\circledR}$ device; and 0.15 for Patient 4, as measured by MPOD module of Multicolor Spectralis $\AA_{\text {. }}$.

\section{Figure 2: MPOD measurement by MPOD module of MultiColor Spectralis ${ }^{\circledR}$, Heidelberg Engineering.}

Blue $(488 \mathrm{~nm})$ and green $(518 \mathrm{~nm})$ autofluorescence image is acquired simultaneously by dual-wavelength cSLO system of the device (A,C). Differential topographic map of $A F$ is calculated by in-built software (Heidelberg Eye Explorer (HEYEX)), and radial density map of MP distribution is created (B, D). The red and purple markers can be moved to provide the relevant averaged MPOD reading for the area of interest. In our patient 0.5 degrees average MPOD (as pointed by the red marker) was 0.15 in the right eye and 0.18 in the left eye. (The 0.5 degrees area of MPOD measurement corresponds to the MPOD measurement area evaluated by QuantifEye ${ }^{\circledR}$ device) 


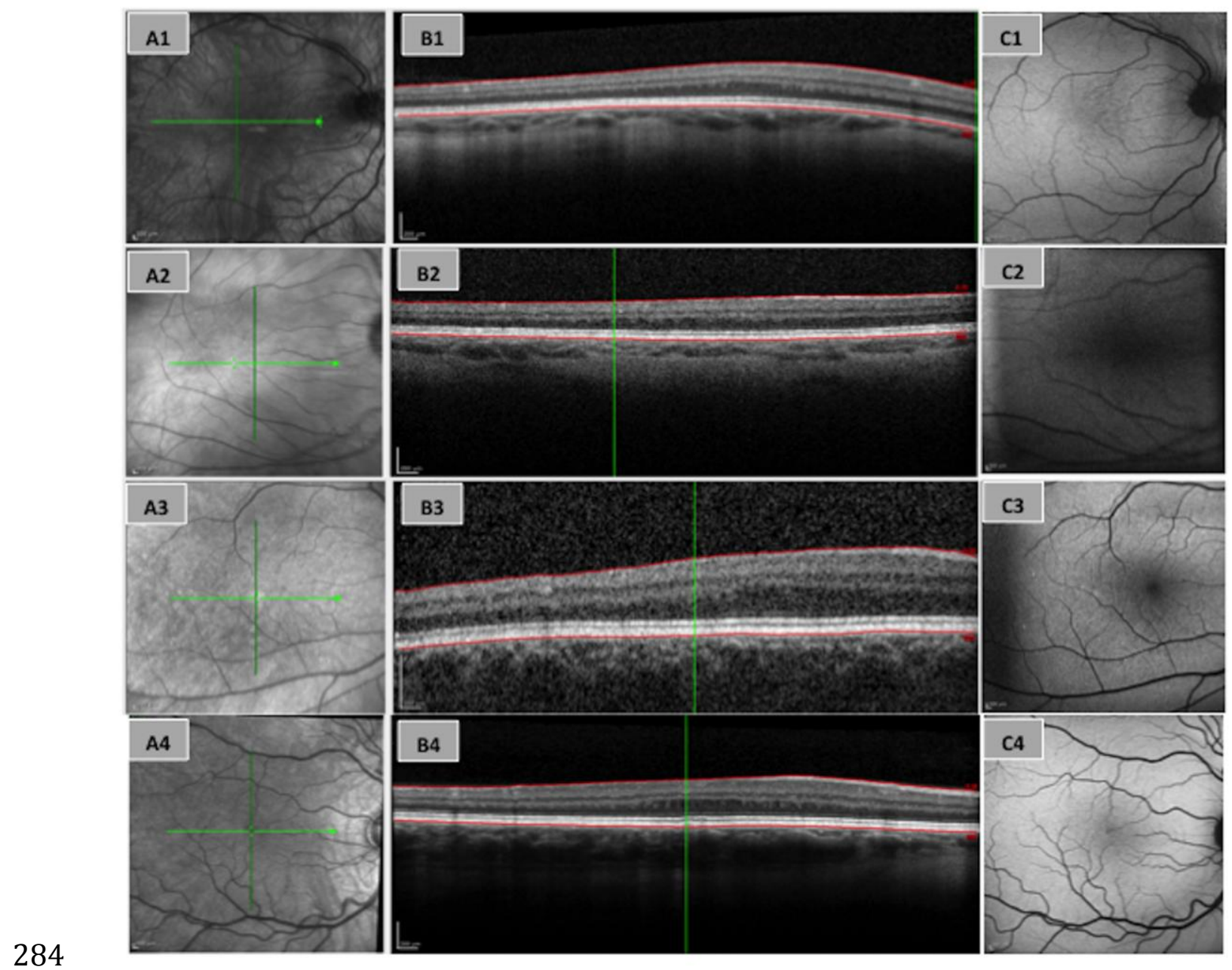

285 Figure 1

286 


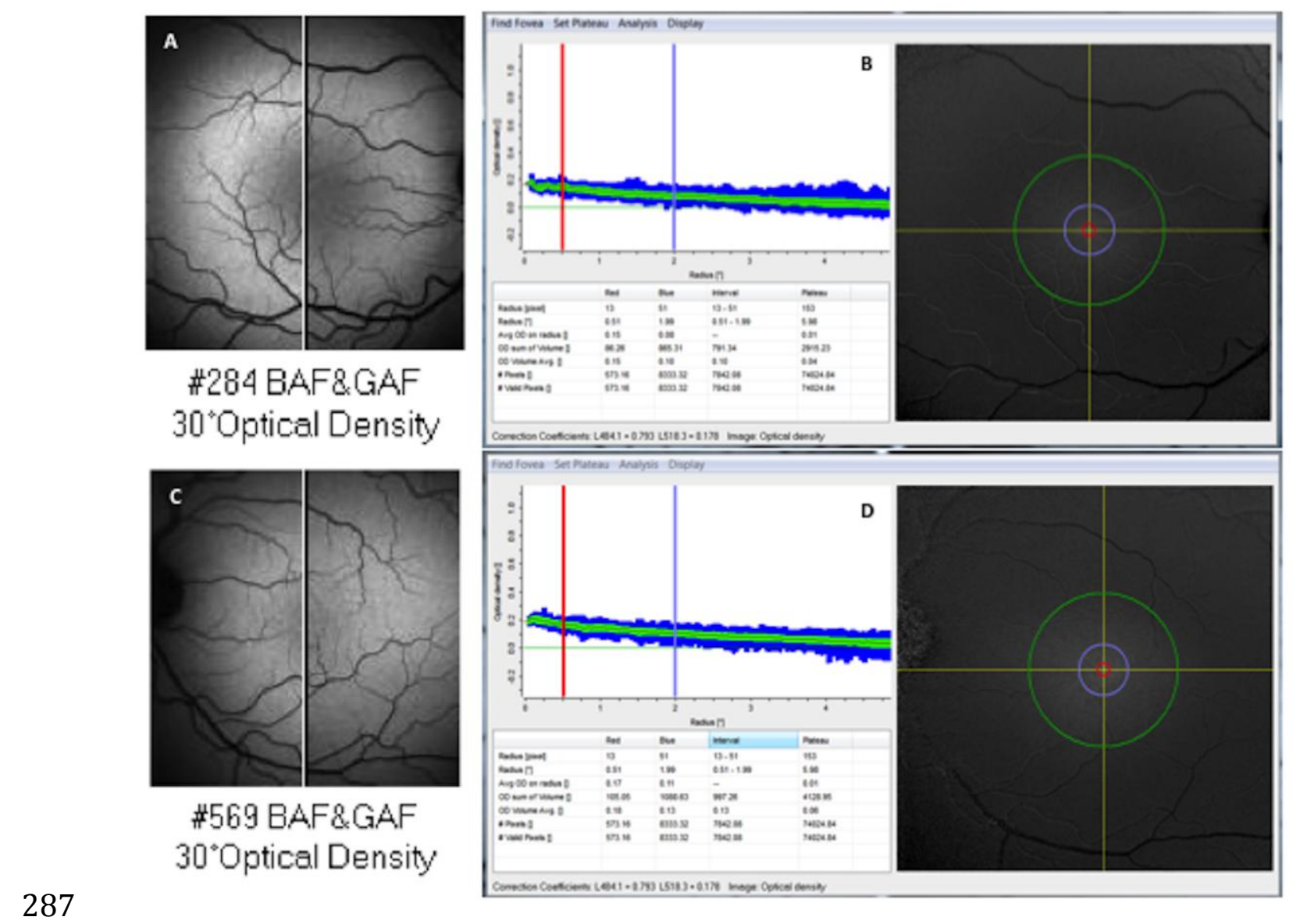

288 Figure 2 